\title{
Modelling Left Atrial Flow and Blood Coagulation for Risk of Thrombus Formation in Atrial Fibrillation
}

\author{
Ahmed Qureshi ${ }^{1}$, Omar Darwish ${ }^{1}$, Des Dillon-Murphy ${ }^{1}$, Henry Chubb ${ }^{1}$, Steven Williams ${ }^{1}$, Dmitry \\ Nechipurenko ${ }^{2}$, Fazoil Ataullakhanov ${ }^{2}$, David Nordsletten ${ }^{1}$, Oleg Aslanidi ${ }^{1}$, Adelaide de Vecchi ${ }^{1}$ \\ ${ }^{1}$ School of Biomedical Engineering and Imaging Sciences, King's College London, United Kingdom \\ ${ }^{2}$ Department of Physics, Lomonosov Moscow State University, Russia
}

\begin{abstract}
Atrial fibrillation (AF) diminishes left atrial (LA) mechanical function and impairs blood flow. The latter can lead to blood stasis and increased risk of thrombus formation and stroke. We investigate this risk by studying the effects of LA flow in sinus rhythm (SR) and AF on blood coagulation dynamics.

Patient-specific computational fluid dynamics (CFD) simulations were coupled with the reaction-diffusionconvection equation for thrombin. Patient LA wall motions driving the flow were reconstructed from Cine MRI data during SR and AF. 15 cardiac cycles were simulated for each patient to evaluate the likelihood of thrombus formation in the critical left atrial appendage (LAA) and right inferior pulmonary vein (RIPV) regions.

The simulations showed that mean blood flow velocity in the LA cavity was substantially decreased (47\%) during AF compared to SR. Specifically in LAA, mean flow velocities decreased from $0.06 \mathrm{~m} / \mathrm{s}$ in $S R$ to $0.035 \mathrm{~m} / \mathrm{s}$ in $A F$, leading to enhanced thrombin generation. In the $R I P V$, higher mean flow velocities $(0.16 \mathrm{~m} / \mathrm{s})$ enabled thrombin washout through the mitral valve irrespective of SR or AF.

This study proposes a novel modelling approach for quantifying the likelihood of AF-related thrombogenesis within LA and demonstrates increased risk of thrombus formation in the LAA when compared with the RIPV.
\end{abstract}

\section{Introduction}

Atrial fibrillation (AF) is the most prevalent cardiac arrhythmia worldwide and is associated with five-fold increased risk of thromboembolic stroke [1]. Irregular electrical activations lead to abnormal atrial mechanical contractions, which in turn alter blood flow within the left atrium (LA), particularly in LA appendage (LAA).

Blood coagulation is believed to be induced through altered blood flow, vascular injury and variations in blood constituents - each linked to AF [2]. Blood interaction with damaged vascular walls initiates the coagulation cascade, which ultimately activates the key clotting protein thrombin to form a stable clot plug and facilitate wound healing. However, thrombus detachment can have dangerous complications such as stroke - one of the leading causes of mortality. Variable morphologies of the LAA make it prone to thrombogenesis and responsible for over $90 \%$ of AF-related thrombi [3]. While sinus rhythm (SR) provides ample blood flow to the LAA, preventing build-up of thrombogenic proteins, reduced LA function in AF increases blood stagnation and a predisposition to thrombus formation [2].

Blood flow and stasis conditions in the LAA can be investigated through computational fluid dynamics (CFD) simulations based on patient imaging data. Patientspecific CFD models have been employed to investigate blood flow in realistic LA geometries, yet few studies have coupled macroscopic flow modelling with models of thrombogenesis [4]. The coupled simulation of blood flow and coagulation dynamics in the LA of AF patients can become a powerful diagnostic tool for tailoring standard, mechanism-based, stroke risk assessment and anticoagulation therapy to the individual patient.

This proof-of-concept study aims to show how patientspecific blood flow conditions can affect thrombus formation and growth in different LA regions.

\section{Methods}

Large-scale CFD models of blood flow were coupled with chemical reaction-diffusion-convection models of blood coagulation dynamics. This approach was first tested in $2 \mathrm{D}$, and then applied to evaluate the generation and accumulation of thrombin in patient-specific 3D LA models, simulating the thrombus formation process. LA regions with fast and slow flow were chosen to compare the impact of blood velocity on coagulation dynamics.

\subsection{Modelling Flow and Coagulation}

Blood velocity, $\boldsymbol{u}$, was calculated by solving the incompressible Navier-Stokes equation, Eq. (1):

$$
\rho\left(\frac{\partial \boldsymbol{u}}{\partial t}+(\boldsymbol{u} \cdot \nabla) \boldsymbol{u}\right)=-\nabla p+\mu \Delta \boldsymbol{u}
$$


Here $\rho=1060 \mathrm{Kg} / \mathrm{m}^{3}$ is blood density and $\mu=$ $3.5 \times 10^{-3} \mathrm{PaS}$ is viscosity. Blood coagulation was modelled by the following reaction-diffusion-convection equation for the thrombin concentration, $\mathrm{Th}\left(\mathrm{mol} / \mathrm{m}^{3}\right)$ :

$$
\frac{\partial T h}{\partial t}=D_{T h} \cdot \Delta T h-\boldsymbol{u} \cdot \nabla T h+R_{T h}
$$

Here $D_{T h}=4.6 \times 10^{-11} \mathrm{~m}^{2} / \mathrm{s}$ is diffusivity. Eq. (3) represents the biochemical reactions of thrombin, with values for constants $K_{1}-K_{5}$ summarised in Table 1 [5]:

$R_{T h}=K_{1}\left(1+K_{2} T h\right)\left[K_{3} T h\left(1+K_{4} T h\right)\right]\left(1-\frac{T h}{u_{0}}\right)-K_{5} T h$

Table 1. Constants, values and units for Eq. (3).

\begin{tabular}{ccc}
\hline Constant & Value & SI Unit \\
\hline$K 1$ & $4.083 \times 10^{-3}$ & $1 / \mathrm{s}$ \\
$K 2$ & $447.66 \times 10^{6}$ & $\mathrm{~m}^{3} / \mathrm{mol}$ \\
$K 3$ & $2.75 \times 10^{-6}$ & - \\
$K 4$ & $4.89 \times 10^{6}$ & $\mathrm{~m}^{3} / \mathrm{mol}$ \\
$K 5$ & $3.83 \times 10^{-2}$ & $\mathrm{~m}^{3} / \mathrm{mol}$ \\
$u_{0}$ & $1.4 \times 10^{-4}$ & - \\
\hline
\end{tabular}

An initial condition of Th $=1 \times 10^{-10} \mathrm{~mol} / \mathrm{m}^{3}$ was applied to specific areas of interest to mimic LA wall damage, while $T h$ was set to zero in the rest of LA.

\subsection{CFD Solver}

For this study, the finite-element-based solver CHeart was used to perform CFD simulations of LA flow [6]. Streamline upwind Petrov-Galerkin (SUPG) stabilisation was applied to control the convection-dominated LA flow, a discontinuity operator resolved steep concentration gradients and stabilised Neumann boundary conditions were set at inlets and outlets to minimise instabilities [7].

\subsection{D Left Atrial Model}

The coupled CFD and thrombin models were first tested on a 2D LA geometry with semi-realistic boundary

(1)

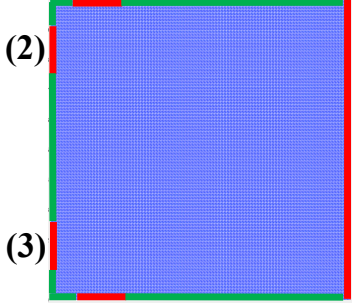

(4)
(5)

Figure 1. 2D LA model with PV inlets, MV outlet (red) and LA wall (green) boundaries. conditions applied on a triangular mesh with $79 \mathrm{k}$ elements (Figure 1) and solved using a time-step of $7.5 \mathrm{~ms}$ for 400 steps (total time $3 \mathrm{~s}$ ). Six boundaries characterised the LA; four inlets (1-4) denoted the pulmonary veins (PVs), one outlet (5) for the mitral valve (MV), and the remaining boundary (6) as the LA wall. A pulsatile flow velocity of $0.5 \mathrm{~m} / \mathrm{s}$ was prescribed at each inlet.

\subsection{D Left Atrial Models}

3D deforming LA volumes were derived from Cine MRI datasets acquired for two patients, one in SR and one during $\mathrm{AF}[4,8]$. The datasets had spatial resolution of $1.4 \times 1.4 \times 10 \mathrm{~mm}^{3}$ and temporal resolution of 50 time phases per cycle acquired on a $1.5 \mathrm{~T}$ Philips Ingenia scanner. The endocardial surface was manually segmented to generate 3D LA models. A feature-tracking algorithm based on temporal sparse free form deformations was applied producing time-dependant, deforming LA meshes corresponding to the 50 phases of the cardiac cycle [9]. The mesh series was then temporally interpolated to create 1000 steps per cycle. Tetrahedral volume meshes ( $400 \mathrm{k}$ elements) were generated and Arbitrary Lagrangian Eulerian (ALE) based simulations were performed using CHeart for 15 cardiac cycles (time-step of $0.73 \mathrm{~ms}$, total time 10.95s). The models simulated LA blood flow and thrombin concentration dynamics, focusing on the fast flowing RIPV and slow flowing LAA regions.

\section{Results}

\subsection{D Left Atrium}
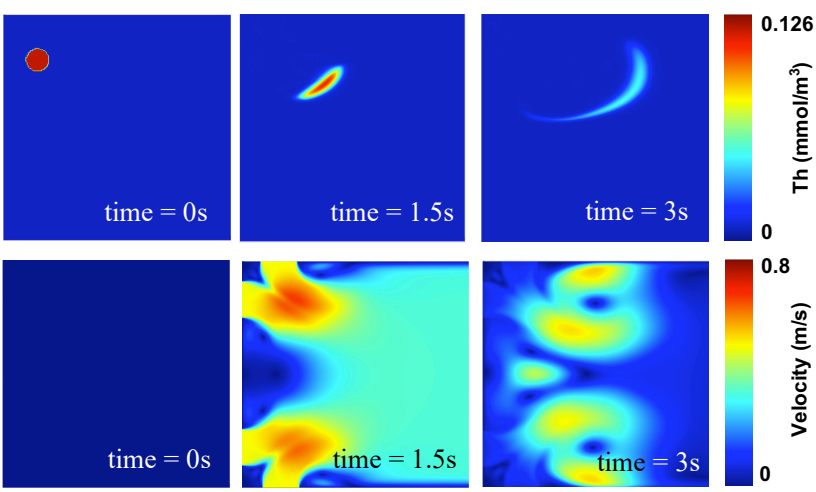

Figure 2. CFD simulations of thrombin concentration and blood velocity for one inflow cycle in the 2D LA.

2D LA flow simulations coupled with the reactiondiffusion-convection equation for thrombin (Figure 2) showed numerical stability throughout the domain and time series. Thrombin concentration had two steady state values, $1.26 \times 10^{-4} \mathrm{~mol} / \mathrm{m}^{3}$ (coagulation) and $0 \mathrm{~mol} /$ 
$m^{3}$ (no coagulation), and hence a threshold value of $9 \times 10^{-5} \mathrm{~mol} / \mathrm{m}^{3}$ was used to define a thrombus.

As blood velocity was increased at the PV inlets, the initial concentration of thrombin (near boundaries $\mathbf{1}$ and 2) began to decrease and diffuse after $1.5 \mathrm{~s}$ and continued to decrease at $3 s$ under the influence of blood velocity. After the first cardiac cycle thrombin concentration in the entire mesh decreased to $0 \mathrm{~mol} / \mathrm{m}^{3}$, attributed to washout through the MV outflow boundary (5).

\subsection{D Left Atrium Models}

The two patient-specific models, for SR and AF, were used to compare the effect of AF on blood coagulation in LA, specifically in the LAA and RIPV regions (Figure 3). In the SR model, mean LA velocity was $0.21 \mathrm{~m} / \mathrm{s}$, which decreased to $0.11 \mathrm{~m} / \mathrm{s}$ in the AF model (47\% reduction). Blood velocity values are summarised in Table 2 .

\subsubsection{Right Inferior Pulmonary Vein}

In the SR model, thrombin in the RIPV showed no build up, with the initial concentration reduced to $0 \mathrm{~mol} / \mathrm{m}^{3}$ in most of the region, with low levels near the MV outflow (Figure 3a). In the AF model, lower flow

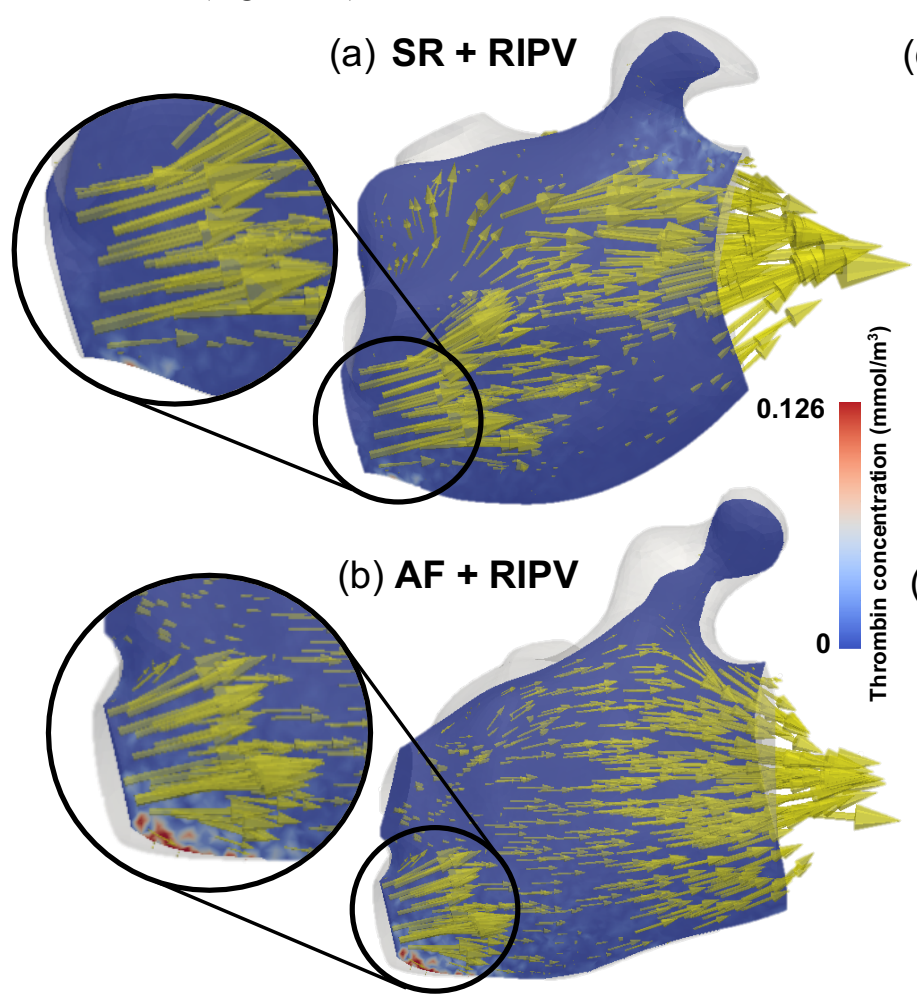

velocities at the RIPV facilitated a buildup of thrombin, albeit in a relatively small region (Figure $3 b$ ).

Table 2. Blood velocities in the LAA and RIPV regions.

\begin{tabular}{c|c|cc}
\cline { 3 - 4 } \multicolumn{2}{c|}{} & Max. Vel. $(\mathrm{m} / \mathrm{s})$ & Mean Vel. $(\mathrm{m} / \mathrm{s})$ \\
\hline \multirow{2}{*}{ RIPV } & SR & 2.2 & 0.21 \\
\cline { 2 - 2 } & AF & 1.3 & 0.11 \\
\multirow{2}{*}{ LAA } & SR & 0.6 & 0.06 \\
\cline { 2 - 2 } & AF & 0.29 & 0.04 \\
\hline
\end{tabular}

\subsubsection{Left Atrial Appendage}

The maximum LAA velocity decreased by $52 \%$, and mean velocity decreased by $33 \%$ from SR to $\mathrm{AF}$, which affected the thrombin concentration (Figure $3 \mathrm{c}$ and $3 \mathrm{~d}$ ). In the SR model, there was limited thrombus growth in the LAA, with low concentrations near the MV. However, in the AF model, the thrombus grew substantially within the LAA, doubling in size between cardiac cycles 5 and 15 , with the concentration reaching the high steady state of $1.26 \times 10^{-4} \mathrm{~mol} / \mathrm{m}^{3}$. Subsequently, a small loss of cohesion from the main thrombus area was seen after 15 cardiac cycles (Figure 3d), which may have potential for thrombus detachment.

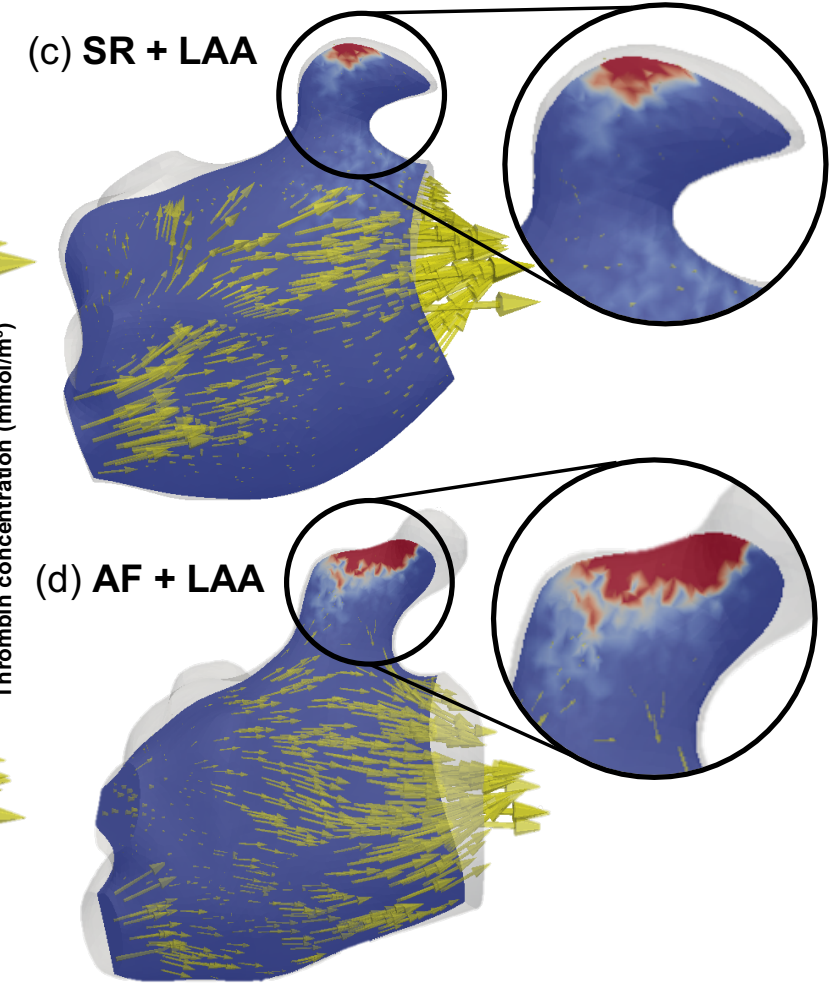

Figure 3. Blood flow and thrombin concentration in LA models during SR and AF, regions of interest highlighted. LA volume slices are shown at peak flow during $15^{\text {th }}$ cardiac cycle, the colour scale represents thrombin concentration while yellow arrows depict the instantaneous blood velocity field. (a) In SR, fast flow in the RIPV region washes away thrombin through MV. (b) In AF, some build-up of thrombin in the RIPV region is seen. (c) In SR, low velocity in LAA facilitates thrombin accumulation. (d) In AF, thrombus grows substantially in LAA where flow is slowest. 


\section{Discussion}

This proof-of-concept study introduces a novel computational modelling workflow for investigating the effect of blood flow velocity on coagulation dynamics and thrombogenesis in the LA of two patients in SR and AF.

2D simulations of the coupled flow and reactiondiffusion-convection model in CHeart were essential for assessing the numerical stability of the equations and applying stabilisation techniques, as described in Section 2.2 [7]. The 2D findings provided the basis for detailed simulations in 3D LA.

3D patient-specific results provided insights into AFrelated thrombogenesis. Flow velocities were in agreement with values from previous studies in 3D cardiac models and 4D flow MRI $[4,10]$. The effect of fast flow on blood coagulation were seen in the RIPV region, with little-to-no buildup of thrombin concentration. The rapid washout of thrombin through the MV as blood enters the PVs suggests a low risk of thrombi forming in the LA cavity. On the other hand, low blood velocity was associated with the thrombus growth seen in LAA, especially during AF. These simulations can provide an explanation to the increased risk of thromboembolic stroke in AF compared to SR, a finding commonly reported in literature [3].

These results help dissect the mechanisms of thrombus formation in the LAA, rather than in the LA cavity [3]. Increased blood stasis in the LAA during AF means no thrombin wash-out, with the thrombin concentration reaching the high steady state of $1.26 \times 10^{-4} \mathrm{~mol} / \mathrm{m}^{3}$. As a result, the accumulation of thrombin in a large area near the LA wall can lead to the generation of a thrombus and dangerous complications in the form of stroke.

Main limitations of the study include a small number of patient cases and simplicity of the coagulation model. Currently, the thrombus is simulated as a continuously growing area of liquid blood with thrombin. In future, addition of variables accounting for fibrinogen and fibrin in the blood coagulation model will enable representing a more realistic, solid thrombus structure. This can be achieved by introducing the fibrin/fibrinogen-dependent Brinkman term to the Navier-Stokes equations, which significantly increases blood viscosity in the thrombus. The model can also be linked to other types of MRI data and atrial electrophysiology underlying AF [11].

\section{Conclusion}

Accurate modelling of 3D blood flow with coagulation dynamics can explain the patient-specific mechanisms of thrombus generation and movements in the blood stream with the potential to improve current stroke risk assessment and anticoagulation therapy for AF patients.

\section{Acknowledgments}

This research was supported by the EPSRC [EP/R513064/1].

\section{References}

[1] P. A. Wolf, R. D. Abbott, and W. B. Kannel, "Atrial fibrillation as an independent risk factor for stroke: The framingham study," Stroke, vol. 22, no. 8, pp. 983 988, 1991.

[2] W. Y. Ding, D. Gupta, and G. Lip, "Atrial fibrillation and the prothrombotic state: revisiting Virchow's triad in 2020," Heart, p. heartjnl-2020-316977, 2020.

[3] J. L. Blackshear and J. A. Odell, "Appendage obliteration to reduce stroke in cardiac surgical patients with atrial fibrillation," Annals of Thoracic Surgery, vol. 61, no. 2. Elsevier USA, pp. 755-759, 1996.

[4] D. Dillon-Murphy et al., "Modeling left atrial flow, energy, blood heating distribution in response to catheter ablation therapy," Front. Physiol., vol. 9, p. 1757, Dec. 2018.

[5] F. I. Ataullakhanov, V. I. Zarnitsyna, A. Y. Kondratovich, E. S. Lobanova, and V. I. Sarbash, "A new class of stopping self-sustained waves: a factor determining the spatial dynamics of blood coagulation," Physics-Uspekhi, vol. 45, no. 6, pp. 619636, Jun. 2002.

[6] J. Lee et al., "Multiphysics computational modeling in CHeart," SIAM J. Sci. Comput., vol. 38, no. 3, pp. C150-C178, 2016.

[7] S. R. Lynch et al., "Numerical considerations for advection-diffusion problems in cardiovascular hemodynamics," Int J Numer Meth Biomed Engng. 2020; 36:e3378.

[8] H. Chubb et al., "The reproducibility of late gadolinium enhancement cardiovascular magnetic resonance imaging of post-ablation atrial scar: a crossover study," J. Cardiovasc. Magn. Reson., vol. 20, no. 1, p. 21, Dec. 2018

[9] E. Kerfoot et al., "Eidolon: Visualization and computational framework for multi-modal biomedical data analysis." LNCS, Vol. 9805 (Springer), 425-437

[10] J. U. Fluckiger et al., "Left atrial flow velocity distribution and flow coherence using four-dimensional flow MRI: A pilot study investigating the impact of age and pre- and postintervention atrial fibrillation on atrial hemodynamics," J. Magn. Reson. Imaging, vol. 38, no. 3, pp. 580-587, Sep. 2013.

[11] A. Roy, M. Varela, and O. Aslanidi, "Image-based computational evaluation of the effects of atrial wall thickness and fibrosis on re-entrant drivers for atrial fibrillation.," Front. Physiol., vol. 9, p. 1352, 2018.

\section{Address for correspondence:}

Ahmed Qureshi; Biomedical Engineering and Imaging Sciences Department, King's College London, $3^{\text {rd }}$ Floor Lambeth Wing, St Thomas' Hospital, London, SE1 7EH, UK; E-mail:ahmed.qureshi@kcl.ac.uk 